\title{
Electrostatic and Hydrophobic Interactions in NaCMC Aqueous Solutions: Effect of Degree of Substitution
}

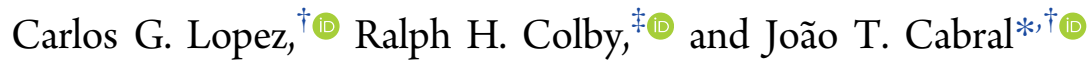

${ }^{\dagger}$ Department of Chemical Engineering, Imperial College London, London SW7 2AZ, U.K.

${ }^{\ddagger}$ Department of Materials Science and Engineering, The Pennsylvania State University, University Park, Pennsylvania 16802, United States

Supporting Information

ABSTRACT: The rheology of water-soluble polyelectrolytes at intermediate and high concentrations is controlled by entanglement, hydrophobic, and electrostatic interactions, whose influences are difficult to isolate. We investigate the rheology of semidilute solutions of sodium carboxymethyl cellulose $(\mathrm{NaCMC})$ with molecular weight $M_{\mathrm{w}} \simeq 2.5 \times 10^{5} \mathrm{~g} /$ mol and varying degree of substitution (DS) as a function of polymer concentration in various solvent media: salt-free water (long-ranged electrostatic interactions), $0.5 \mathrm{M}$ aqueous $\mathrm{NaCl}$
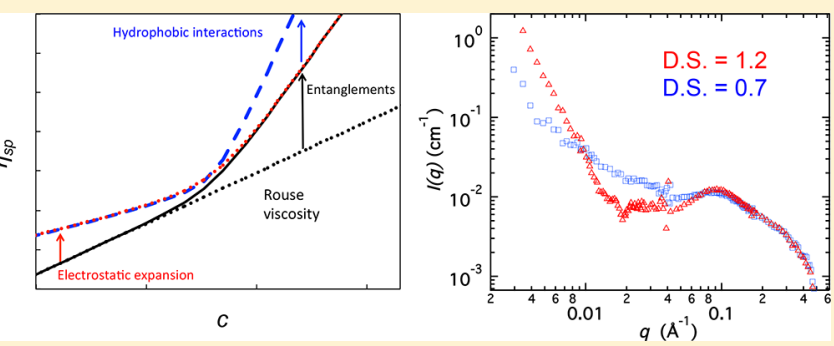
(screened electrostatics), and $0.5 \mathrm{M}$ aqueous $\mathrm{NaOH}$ (screened electrostatics, diminished hydrophobic interactions) in order to selectively examine the role played by these different interactions. Decreasing DS is found to decrease solubility and induce partial aggregation and eventual gelation. In salt-free and $0.5 \mathrm{M} \mathrm{NaCl}$ solution, $\mathrm{NaCMC}$ with $\mathrm{DS} \simeq 1.2$ exhibits hydrophilic polyelectrolyte and neutral polymer in good solvent behavior, respectively. Decreasing DS to $\simeq 0.7-0.8$ leads to hydrophobic behavior in both media, becoming weak gels at high concentrations. In $0.5 \mathrm{M}$ $\mathrm{NaOH}(\mathrm{pH}=13.5)$ the viscosities of solutions with different DS become identical when plotted against the overlap parameter, which we interpret as resulting from the solubilization of unsubstituted cellulose blocks. Small-angle neutron scattering (SANS) data indicate that the polymer conformation is not strongly affected by hydrophobic interactions. By varying DS, ionic strength, and $\mathrm{pH}$, we demonstrate the tuning of $\mathrm{NaCMC}$-solvent interactions, controlling separately the electrostatic and hydrophobic effects on the solution rheology.

\section{INTRODUCTION}

Water-soluble polyelectrolytes play a crucial role as rheology modifiers, stabilizers, and functional ingredients in a range of formulations. ${ }^{1}$ Significantly, electrostatic forces promote solution stability and result in expanded polymer conformations. Further, hydrophobic groups on the polymer chains promote transient interchain associations, ${ }^{2}$ which lead to enhanced viscosities, ${ }^{3}$ longer relaxation times, ${ }^{4-9}$ aggregate formation, ${ }^{10-13}$ and conformational transitions. ${ }^{14-16}$

While cellulose, the most abundant polymer on earth, ${ }^{17}$ is insoluble in water (and most aqueous salt solutions, including $\mathrm{NaCl}$ ), several of its derivatives may be dissolved at a molecular or colloidal level. ${ }^{18}$ Sodium carboxymethyl cellulose (NaCMC) is an anionic, weak, semiflexible, water-soluble polyelectrolyte widely used as a thickener and rheology modifier. ${ }^{19-23}$ The degree of substitution (DS, the number of carboxymethyl groups per glucose unit) of $\mathrm{NaCMC}$ is defined in Scheme 1 and usually varies between 0.7 and 1.2 in commercial materials, which qualify them as strongly charged polyelectrolytes. ${ }^{19}$

Weakly substituted NaCMC generally exhibit hydrophobic interactions in aqueous solution due to the presence of unsubstituted cellulose blocks, for which water is a nonsolvent. ${ }^{24,25}$ Addition of $\mathrm{NaOH}$ is known to promote unsubstituted cellulose solubilization in aqueous solution and
Scheme 1. Structure of $\mathrm{NaCMC}^{a}$

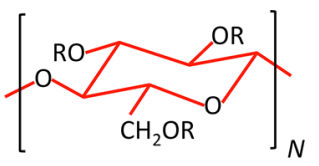

${ }^{a} \mathrm{R}=\mathrm{H}$ or $\mathrm{CH}_{2} \mathrm{COONa}$. The degree of substitution (DS) is the average number of $\mathrm{CH}_{2} \mathrm{COONa}$ groups per monomer, out of a maximum of 3 . The monomer length is $b \simeq 0.515 \mathrm{~nm}$, and the degree of polymerization is $N$.

is therefore expected to remove hydrophobic interactions in weakly substituted NaCMC.

Despite a large number of experimental investigations on the $\mathrm{NaCMC} /$ water/salt solutions (summarized in the next section), the degree of solubility of $\mathrm{NaCMC}$ in semidilute solution and the influence of different interactions (entanglement, electrostatic, hydrophobic) on the flow behavior of $\mathrm{NaCMC}$ solutions are not yet fully resolved.

Received: January 25, 2018

Revised: $\quad$ March 28, 2018

Published: April 11, 2018 
In this article, we study the rheology of $\mathrm{NaCMC}$ solutions of varying degree of substitution ( $\mathrm{DS}=0.7,0.8$, and 1.2 ) as a function of polymer concentration, from approximately the overlap concentration $\left(c^{*}\right)$ to $100 c^{*}$. All three DS have an effective charge spacing set by the Bjerrum length of $\simeq 0.71 \mathrm{~nm}$ in DI water estimated from conductivity data in semidilute solution. ${ }^{26}$ Three solvent media are considered to systematically screen different interactions: (i) DI water $(\mathrm{pH} \simeq 7)$ for nonscreened interactions, (ii) $0.5 \mathrm{M} \mathrm{NaCl}(\mathrm{pH} \simeq 7)$ providing excess salt, and thus screening of electrostatic forces, and (iii) $0.5 \mathrm{M} \mathrm{NaOH}(\mathrm{pH} \simeq 13.5)$, for which electrostatic interactions are screened and unsubstituted cellulose is solubilized, hence diminishing both electrostatic and hydrophobic interactions. Polymer chains and counterions contribute to the screening of electrostatic, excluded volume, and hydrodynamic forces in all solvent media. The relative strength of these interactions is therefore not only a function of the solvent choice but also polymer conformation. All NaCMC samples are fully neutralized, and hence we do not expect significant further charging of the carboxymethyl groups at $\mathrm{pH}=13.5$ compared to $\mathrm{pH}=7$ aqueous media. ${ }^{27}$ Further, at high $\mathrm{pH}$, the $\mathrm{OH}$ groups in the cellulose backbone are not expected to increase the linear charge density of the polyion either. We employ a combination of rheological and small-angle neutron scattering (SANS) measurements to rationalize the interplay between network conformation, solubility, and flow response in these model conditions.

\section{BACKGROUND THEORY AND REVIEW OF NaCMC SOLUTION RHEOLOGY}

Polymer solutions are classified into three concentration regimes: dilute, semidilute, and concentrated. In dilute solution, chains do not overlap and chain dimensions vary as $R \propto N^{\nu}$, where $N$ is the degree of polymerization and $\nu$ is the Flory exponent. The overlap concentration $c^{*}$ marks the crossover to semidilute solution:

$$
c^{*} \simeq \frac{N}{R^{3}}=\frac{B^{3}}{b^{3} N^{3 \nu-1}}
$$

where $b$ is the monomer length; $B$ is the ratio of the chain's end-to-end distance in dilute salt-free solution to the fully stretched chain length, ${ }^{21,28}$ which is found to be $B \simeq 1$ for $\mathrm{NaCMC}$ and other semiflexible polyelectrolytes, consistent with the fact that the persistence length ${ }^{27}$ for $\mathrm{NaCMC}$ with $0.75<\mathrm{DS}<1.25$ is far larger than $b=0.515 \mathrm{~nm} .^{21,29,30}$ In salt solutions, $c^{*}$ can be estimated as the reciprocal of the intrinsic viscosity, obtained from the Huggins equation. For salt-free solutions the Huggins equation does not apply, ${ }^{31}$ and the estimate $\eta_{\mathrm{sp}}\left(c^{*}\right)=1^{20,32}$ can be used instead. Above $c^{*}$ chains overlap, and excluded volume (and hydrodynamic) interactions are screened for length scales larger than the correlation length:

$$
\xi \simeq R\left(c^{*}\right)\left(c / c^{*}\right)^{-\nu /(3 \nu-1)} \quad c>c^{*}
$$

which reduces to $\xi=(B / b c)^{1 / 2}$ in salt-free solution $(\nu=1)$. The specific viscosity and relaxation times of the solution can be described by the Rouse model:

$$
\begin{aligned}
& \tau_{\mathrm{R}} \simeq \frac{\eta_{\mathrm{s}}\left[R\left(c^{*}\right)\right]^{3}}{k_{\mathrm{B}} T}\left(c / c^{*}\right)^{(2-3 \nu) /(3 \nu-1)} \quad c^{*}<c<c_{\mathrm{e}} \\
& \eta_{\mathrm{R}} \simeq \eta_{\mathrm{sp}}\left(c^{*}\right)\left(c / c^{*}\right)^{1 /(3 \nu-1)} \quad c^{*}<c<c_{\mathrm{e}}
\end{aligned}
$$

where the specific viscosity is $\eta_{\mathrm{sp}}=\left(\eta-\eta_{\mathrm{s}}\right) / \eta_{\mathrm{s}}, \eta$ is the viscosity of the solution at zero shear rate, and $\eta_{\mathrm{s}}$ is that of the solvent. Equation 4 reduces to $\eta_{\mathrm{sp}}=N\left(c b^{3}\right)^{1 / 2} B^{-3 / 2}$ in salt-free solution. Above the entanglement concentration $\left(c_{\mathrm{e}}\right)$, the longest relaxation time and viscosity vary as

$$
\begin{aligned}
& \tau \simeq \tau_{\mathrm{R}}\left(c_{\mathrm{e}}\right)\left(\frac{c}{c_{\mathrm{e}}}\right)^{3(1-\nu) /(3 \nu-1)} \quad c>c_{\mathrm{e}} \\
& \eta_{\mathrm{sp}} ; \eta_{\mathrm{R}}\left(c_{\mathrm{e}}\right)\left(\frac{c}{c_{\mathrm{e}}}\right)^{3 /(3 \nu-1)} \quad c>c_{\mathrm{e}}
\end{aligned}
$$

Eqs 5 and 6 are the scaling predictions for entangled solutions of flexible polyelectrolytes with and without salt present. Polyelectrolytes with added salt are analogous to neutral polymers in good solvent, for which eqs 5 and 6 also apply. For neutral polymers in theta solvent, which is not considered here, a different scaling applies ${ }^{33}$ in entangled solutions.

Excluded volume interactions become fully screened at $c_{\mathrm{D}}$, the crossover to the concentrated regime. Polyelectrolytes and neutral polymers are then expected to show similar conformational and rheological behavior, which is to some extent observed. $^{34-38} c_{\mathrm{D}}$ should correspond to the concentration at which $\xi$ reaches the value of the thermal blob or intrinsic persistence length $\left(l_{0}\right)$ and therefore be independent of $M_{\mathrm{w}}$; for $\mathrm{NaCMC}, l_{0}=\xi$ corresponds to a value of $\simeq 0.14 \mathrm{M}$ in salt-free solution. $^{21}$

Experimentally, it has been found that two crossover concentrations exist beyond the semidilute unentangled regime: above a certain concentration $c_{\mathrm{e}}$, a dependence of $\eta_{\mathrm{sp}}$ $\sim N^{1.8} c^{1.5}$ is observed, and above a second crossover concentration $\left(c^{* *}\right), \eta_{\mathrm{sp}} \sim N^{3} c^{3.4}$ is found for $\mathrm{NaCMC}$ in salt-free solutions scales, reminiscent of entangled neutral polymer behavior in good solvent. ${ }^{20}$ While $c^{* *}$ has been widely interpreted as a crossover to the concentrated regime, the $N$ dependence of $c^{* *} \propto N^{-0.6}$ suggests $c^{* *}$ may correspond instead to an entanglement transition. ${ }^{20}$ Both the $\eta_{\text {sp }} \sim N^{1.8} c^{1.5}$ and the $\eta_{\mathrm{sp}} \sim N^{3} c^{3.4}$ regimes disagree with the scaling prediction for entangled, salt-free polyelectrolyte solutions (eq 6). In this study, we opt to identify $c_{\mathrm{e}}$ and $c^{* *}$ solely from the concentration dependence of $\eta_{\mathrm{sp}}$, and it is not at this point clear whether $c_{\mathrm{e}}$ or $c^{* *}$ marks the crossover to the entangled regime. Using a crossover function between $\eta_{\mathrm{sp}} \sim c^{0.5}$ and $\eta_{\mathrm{sp}} \sim$ $c^{3.4}$ yields a value between $c_{\mathrm{e}}$ and $c^{* *}$, detailed in the Supporting Information of ref 20.

Solution Rheology of Hydrophilic NaCMC (DS $\gtrsim 1)$. Salt-free solutions of highly substituted $\mathrm{NaCMC}$ (DS $\gtrsim 1)$ have been found to exhibit typical polyelectrolyte behavior. ${ }^{20,21,39-41}$ A weak, near Fuoss, power law dependence of the viscosity with concentration $\left(\eta_{s p} \sim c^{2 / 3}\right)$ is observed in semidilute unentangled solutions followed by stronger $\left(\eta_{\mathrm{sp}} \sim c^{3.5}\right)$ power law at high concentrations, characteristic of neutral polymer behavior. $^{20,21,41,42}$ The mesh size (or correlation length) has been reported to scale as $\xi \propto c^{-1 / 2}$ in the semidilute regime, ${ }^{21}$ in agreement with eq $2 .^{28,43}$ The absence of thixotropy and gelling indicates that any aggregates or hydrophobic interactions do not significantly affect its macroscopic properties. $^{21}$

Addition of $\mathrm{NaCl}$ has been found to decrease the viscosity of $\mathrm{NaCMC}$ solutions except at high polymer concentrations, ${ }^{20,22}$ for which electrostatic interactions are highly screened by free counterions even in the absence of added salt. In excess salt, the viscosity of entangled NaCMC solutions has been reported ${ }^{1,22}$ 
Table 1. Sample Name, Nominal and Measured Parameters for the Three Samples Used in This Study

\begin{tabular}{|c|c|c|c|c|c|c|c|c|}
\hline \multirow[b]{2}{*}{ sample } & \multicolumn{2}{|c|}{ nominal } & \multicolumn{6}{|c|}{ measured } \\
\hline & DS & $M_{\mathrm{w}}(\mathrm{g} / \mathrm{mol})$ & DS & $M_{\mathrm{w}}(\mathrm{g} / \mathrm{mol})$ & {$[\eta]^{c}\left(\mathrm{M}^{-1}\right)$} & $M_{0}^{d}(\mathrm{~g} / \mathrm{mol})$ & polydispersity & purity (\%) \\
\hline $\mathrm{CMC} 1.2^{a}$ & $1.15-1.45$ & $2.5 \times 10^{5}$ & $1.2 \pm 0.1$ & $3.2 \times 10^{5}$ & 152 & 256 & 3.4 & $\simeq 99.5$ \\
\hline СMC0.8 & $0.80-0.95$ & $2.5 \times 10^{5}$ & $0.8 \pm 0.1$ & $3.1 \times 10^{5, b}$ & 181 & 226 & & \\
\hline СMC0.7 & $0.65-0.90$ & $2.5 \times 10^{5}$ & $0.7 \pm 0.1$ & $2.1 \times 10^{5, b}$ & 133 & 218 & & \\
\hline
\end{tabular}

${ }^{a}$ From ref 20. ${ }^{b}$ Estimated from intrinsic viscosity; see the Supporting Information. ${ }^{c}$ In $0.5 \mathrm{M} \mathrm{NaCl} .{ }^{d}$ Calculated from DS assuming that each carboxylate group has a $\mathrm{Na}^{+}$counterion.
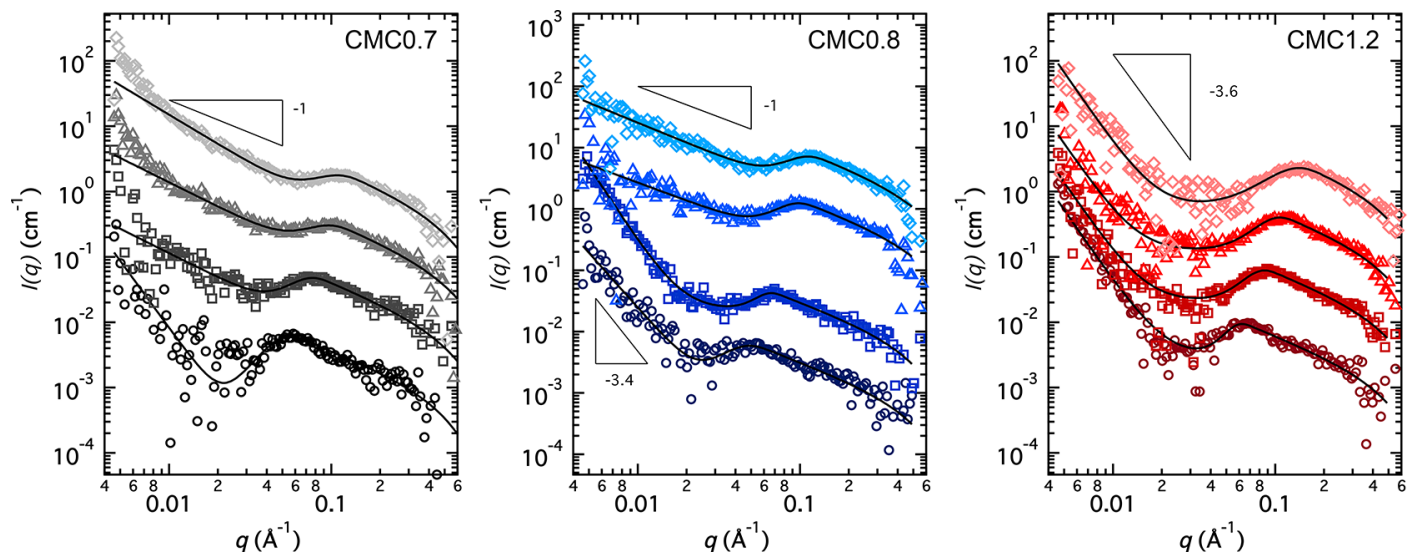

Figure 1. Representative SANS profiles for NaCMC samples in salt-free $\mathrm{D}_{2} \mathrm{O}$, following incoherent background subtraction. CMC0.7 $c \simeq 6,12,20$, and $28 \mathrm{~g} / \mathrm{L}$. Curves are shifted by factors of $1,5,25$, and 125 , respectively, for clarity. CMC0.8 $c \simeq 4,8,20$, and $28 \mathrm{~g} / \mathrm{L}$. Curves are shifted by factors of $1,25,100$, and 500, respectively, for clarity. CMC1.2 $c \simeq 8,16,28$, and $36 \mathrm{~g} / \mathrm{L}$ data ${ }^{21}$ are reported from comparison. Curves are shifted by factors of $1,5,25$, and 125, respectively, for clarity. Lines are descriptive fits as detailed in Supporting Information Figures S2 and S3.

to scale as $\eta_{\mathrm{sp}} \propto N^{3.8} c^{4.1}$, similar to neutral polymers in good solvent and in moderate agreement with eq 6 for $\nu=0.59$.

Solution Rheology of Hydrophobic NaCMC (DS $\lesssim 1$ ). While the average DS has been reported not to significantly affect rheological properties if substitution is random, ${ }^{4-46}$ heterogeneity has been found to lead to stronger exponents of the viscosity with concentration ${ }^{47,48}$ and longer relaxation times. ${ }^{49,50}$

Concentrated solutions of heterogeneous samples are reportedly thixotropic ${ }^{51-55}$ and can form gels. ${ }^{4-48,56-60}$ Fuller and co-workers ${ }^{61}$ inferred from rheo-optical measurements that for $\mathrm{DS} \leq 1$ semidilute and concentrated $\mathrm{NaCMC}$ solutions are highly aggregated.

Elliot and co-workers, ${ }^{45,51}$ Francis ${ }^{44}$ (DS $\simeq 0.7$ ), and Enebro et al. $^{49}(D S \simeq 1)$ reported that samples with similar average degree of substitution but different regularity show markedly different properties. Concentrated solutions (1-3 wt \%, $M_{\mathrm{w}} \simeq$ $\left.1.5 \times 10^{5} \mathrm{~g} / \mathrm{mol}^{62,63}\right)$ of "blocky" samples exhibited thixotropy and crystallinity, while the regularly substituted ones only showed pseudoplasticity. After centrifugation, the obtained gel phase displayed highly thixotropic flow while the solution phase displayed nonthixotropic pseudoplastic flow. These observations suggest that unsubstituted regions, acting as temporary cross-links, are the cause of thixotropy and gelation. 45,46 Francis ${ }^{44}$ and Yang ${ }^{64}$ reported that the viscosity of $\mathrm{NaCMC}$ aqueous salt solutions depends on whether the polymer or the salt is dissolved first, the viscosity being higher in the former case, and the effect was found to be stronger for lower DS samples.

Equations 3-6 do not take into account attractive interactions between polymer chains, ${ }^{65,66}$ which lead to stronger power law exponents for the concentration dependence of the viscosity and relaxation times ${ }^{66}$ and may cause a dependence of $\eta_{\mathrm{sp}} \sim \exp \left(c^{\alpha}\right)$, with $\alpha \simeq 0.6^{65}$ In the following, we will apply eqs $2-6$ to the data presented while, for completion, the Supporting Information considers the possible exponential dependences of the viscosity which may arise from attractive interactions (Figure S10).

\section{METHODS}

The main characteristics of the three NaCMC samples (SigmaAldrich) investigated are summarized in Table 1. The DS of the samples was measured by converting the polymer into its acid form, dissolving it in aqueous $\mathrm{NaOH}$, and titrating the excess $\mathrm{NaOH}$ with $\mathrm{HCl}$ using phenolphthalein as an indicator. The characterization of sample CMC1.2 was reported previously. ${ }^{20,21}$ Water was obtained from a Milli-Q source (resistivity of $18 \mu \mathrm{S} / \mathrm{cm})$, and $\mathrm{D}_{2} \mathrm{O}(99.8 \% \mathrm{D}$ content) was purchased from Cambridge Isotopes. SANS experiments were carried out at SANS2D (ISIS pulsed source, U.K.) with a scattering wavenumber $(q)$ range of $0.0045-0.8 \AA^{-1}$ and at D22 (ILL, France) with $\lambda=6 \AA$ and sample-detector distances of 1.5, 5.6, and $17.6 \mathrm{~m}$ yielding a $q$ range of $0.003-0.6 \AA^{-1}$. Quartz Hellma cells of path length 5 or $2 \mathrm{~mm}$ were employed, depending on polymer concentration.

Rheological measurements were carried out on two stresscontrolled rheometers: a TA hybrid using a cone and plate geometry of diameter 40 or $60 \mathrm{~mm}$ and angle of $1^{\circ}$ or $2^{\circ}$ and a Malvern Kinexus pro with a $40 \mathrm{~mm}$, angle $1^{\circ}$, and cone and plate geometry. Selected viscosity measurements were carried out on a LV-DVI-Prime Brookfield viscometer with a Couette geometry, at a shear rate lower than the onset of shear thinning.

\section{RESULTS AND DISCUSSION}

Small-Angle Neutron Scattering (SANS). Figure 1 shows representative SANS profiles for different concentrations of samples CMC0.7, CMC0.8, and CMC1.2 in salt-free $\mathrm{D}_{2} \mathrm{O}$ solutions. A correlation peak at wavenumber $q^{*}$ is clear for all samples at all concentrations. The value of $q^{*}$ is determined as 
the local maximum in the scattering intensity. The scattering intensity shows a $I \propto q^{-1}$ dependence for $q>q^{*}$ as expected for polyelectrolytes in salt-free water $(\nu=1)$, while a stronger dependence seen at very high $q$ arises from the finite lateral dimensions of the chain, with a radius of $r_{\mathrm{p}} \simeq 3-4 \AA \AA^{21}$ Details of the fitting procedure are provided in the Supporting Information (Figures S1-S4). The peak position as a function of concentration for the three samples studied, in salt-free water, is shown in Figure 2. A power law of $q^{*} \propto c^{1 / 2}$ is

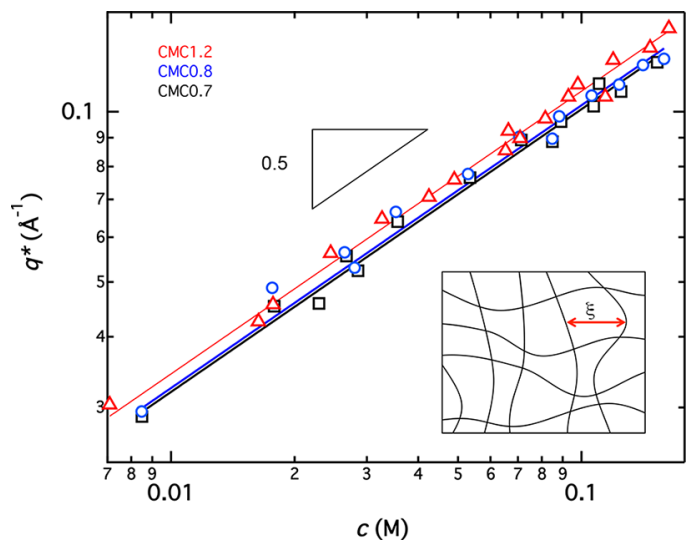

Figure 2. Peak position for $\mathrm{CMC} 1.2(\triangle$, data from ref 21$)$, CMC0.8 $(\mathrm{O})$, and $\mathrm{CMC0.7}(\square)$ as a function of polymer concentration. Lines are best fits to a power law with exponent of $1 / 2$.

observed for all samples, consistent with eq 2 for $\nu=1(\xi=2 \pi)$ $\left.q^{*}\right)$. We do not expect a local collapse at high concentrations, as observed for hydrophobic flexible polyelectrolytes due to the relatively high intrinsic rigidity of the NaCMC chains. ${ }^{8,9,67}$

The correlation length of flexible polyelectrolyte solutions has been reported to vary with charge density. ${ }^{68}$ This occurs because as the charge density increases, the electrostatic blob $\left(\xi_{\mathrm{T}}\right)$ decreases in size, leading to chain unfolding locally. Fitting eq 2 to the data presented in Figure 2 yields $B \simeq 1.1 \pm 0.1$ for sample $\mathrm{CMC} 1.2$ and $B \simeq 1.2 \pm 0.1$ for samples $\mathrm{CMC} 0.8$ and CMC0.7 as expected for locally rigid polyelectrolytes. Since it is unlikely that $\mathrm{NaCMC}$ can fold locally given its intrinsic persistence length of $\simeq 5 \mathrm{~nm}$ (or 10 monomers), it is possible that the marginal increase in $B$ with decreasing DS results from a smaller number of chains contributing to the network. This may occur due to partial lateral aggregation for example. However, within experimental error, we cannot rule out that chains are fully soluble and no conformational change occurs in the DS range studied. Taking only the high concentration regime $(c \gtrsim 0.09 \mathrm{M})$ and assuming the CMC1.2 sample is fully soluble, we can calculate the percentage of chains not contributing to the mesh size to be $\simeq 10-15 \%$ for the CMC 0.8 and CMC0.7 samples. This result indicates that most chains are molecularly dispersed for the weakly substituted samples, with a small fraction possibly aggregated and/or collapsed.

An upturn at low $q$, characteristic of polyelectrolyte solutions ${ }^{9,69-73}$ is also observed. Low $q$ power laws have been speculated to be related to the mesoscopic clustering of polyelectrolyte solutions, whose dynamic origin remains controversial. $^{72,74-77}$ For CMC1.2, a power law of $I(q) \propto$ $q^{-3.6}$, independent of polymer concentration was found in an earlier study. ${ }^{21} \mathrm{CMC} 0.7$ and $\mathrm{CMC} 0.8$ show similar behavior at low concentrations $(c \lesssim 10-15 \mathrm{~g} / \mathrm{L})$. At high concentrations, a weaker power law of $I(q) \propto q^{-1}$ is observed, which could be related to the formation of fringed micelles. ${ }^{18,46,78-81}$ These supramolecular structures, which are common for cellulose derivatives, consist of a core of laterally aggregated (perhaps crystalline) chains, out of which dangling chains emanate. This hypothesis is consistent with the results for the correlation length outlined above. Fitting the low $q$ upturn is challenging because of the large number of free parameters required to describe fringed micelle aggregates and the added difficulty of fitting both a polydisperse population of aggregates and a soluble chain polyelectrolyte mesh simultaneously over a limited $q$ range (as detailed in Figure S4).

In summary, SANS data indicate that in salt-free $\mathrm{D}_{2} \mathrm{O}$ aCMC is molecularly dispersed with an extended local conformation for the DS range investigated in this study. We next report the rheological properties of these samples to understand the effect of different interactions on their flow behavior.

Steady Shear Rheology. Viscosity data as a function of shear rate were fitted to the Carreau model:

$$
\eta(\dot{\gamma})=\frac{\eta(0)}{\left(1+(\dot{\gamma} \tau)^{2}\right)^{n / 2}}
$$
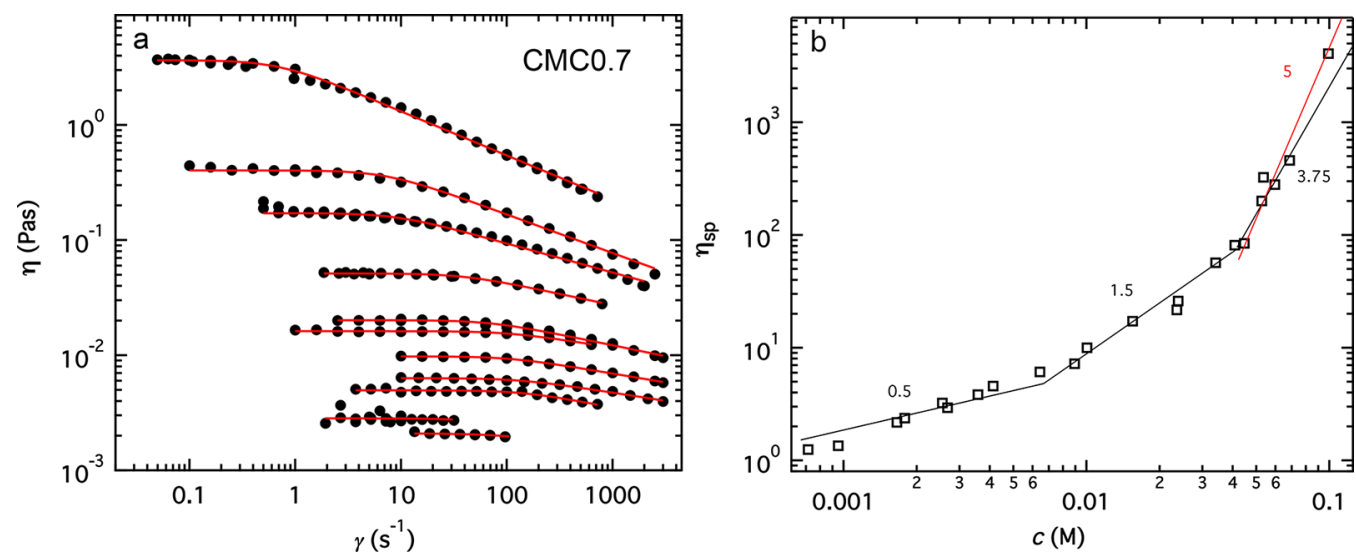

Figure 3. Rheology of CMC0.7 salt-free aqueous solutions. (a) Viscosity as a function of shear rate for selected samples of varying concentration along with fits to the Carreau model (eq 7 with $b=n$ ) (red lines). The concentrations are: 0.00098, 0.0017, 0.0042, 0.0066, 0.01, 0.016, 0.024, 0.035, $0.054,0.07,0.1 \mathrm{M}$. (b) Specific viscosity at zero shear rate as a function of concentration. Black lines are scaling theory predictions; the dashed red line is the best fit power law for $c>0.04 \mathrm{M}$. 
where $\eta(0)$ is the zero shear rate viscosity, $\dot{\gamma}$ is the shear rate, $\tau$ is the relaxation time, and $n$ the power law exponent of the apparent viscosity in the shear thinning regime. For samples that did not exhibit shear thinning, an average over all viscosity values was taken as $\eta(0)$. Fits to the Cross and the CarreauYasuda models (the Carreau model is a particular case of the latter) are considered in the Supporting Information (Figures S5-S8). The different models for the shear rate dependent viscosity yield similar values of $\eta(0)$, but different values for $\tau$ and $n$. The generalized Carreau-Yasuda model fits the data well at all concentrations but the estimates of $\tau$ are less reliable due to the additional free parameter required. The Cross model fits the data well, but it gives values of $\tau$ and $n$ which differ significantly from other models as well as from model-free estimates of these parameters. We have opted to use the Carreau model as it provides a reliable estimate of the different flow properties with a limited number of fit parameters.

Salt-Free Solutions. Representative viscosity data as a function of shear rate along with fits to the Carreau model for sample CMC0.7 in D.I. water are plotted in Figure 3a. The concentration dependence of the specific viscosity at zero shear rate is plotted in Figure $3 \mathrm{~b}$, along with the scaling predictions for the semidilute unentangled $\left(\eta_{\mathrm{sp}} \propto c^{1 / 2}\right)$, semidilute entangled $\left(\eta_{\mathrm{sp}} \propto c^{3 / 2}\right)$, and concentrated $\left(\eta_{\mathrm{sp}} \propto c^{15 / 4}\right)$ regimes. Table 2 compiles $c^{*}$ and $c_{\mathrm{e}}$ (calculated from the onset of the $\eta_{\mathrm{sp}}$ $\propto c^{3 / 2}$ dependence) and $c^{* *}$ (calculated from the onset of the $\eta_{\mathrm{sp}} \propto c^{15 / 4}$ dependence) for the different samples studied.

Table 2. Overlap and Entanglement Concentrations for the Three Samples Studied in Salt-Free Solution ${ }^{a}$

$\begin{array}{cclccc}\text { sample } & c^{*}(\mathrm{M}) & c_{\mathrm{e}}(\mathrm{M}) & c^{* *}(\mathrm{M}) & c_{\mathrm{e}} / c^{*} & c^{* *} / c_{\mathrm{e}} \\ \text { CMC1.2 } & 0.00012 & 0.011 & 0.055 & 92 & 5 \\ \text { CMC0.8 } & 0.00016 & 0.0075 & 0.039 & 47 & 5 \\ \text { CMC0.7 } & 0.00027 & 0.0067 & 0.045 & 25 & 7\end{array}$

${ }^{a} c_{\mathrm{e}}$ calculated from the crossover between $\eta_{\mathrm{sp}} \propto c^{0.5}$ to $\eta_{\mathrm{sp}} \propto c^{1.5}$ and $c^{* *}$ from the crossover between $\eta_{\mathrm{sp}} \propto c^{1.5}$ to $\eta_{\mathrm{sp}} \propto c^{3.75}$. See Figure 3 for the determination of these concentrations for sample CMC0.7. The values for CMC1.2 were determined in ref 21 (see Figure 2).

The stretching parameter $B$ may be calculated from viscosity data in the unentangled regime using eq $4\left(B_{\eta}=\right.$ $\left.\eta_{\mathrm{sp}}{ }^{-2 / 3} N^{2 / 3}\left(c b^{3}\right)^{1 / 3}\right)^{82}$ or from correlation length data using eq $2\left(B_{\xi}=b c \xi^{2}\right)$ obtained from SANS. Figure 4 compares $B_{\xi}$ and

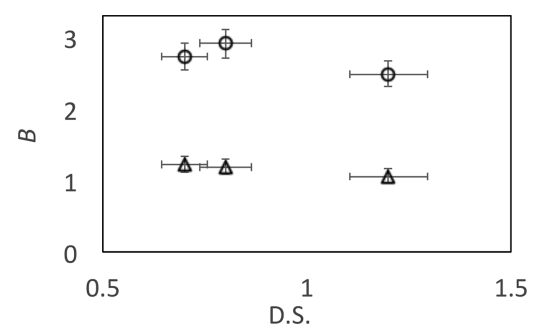

Figure 4. Stretching parameter $B$ as a function of DS, estimated from $(O)$ viscosity data, $B_{\eta}$, and $(\triangle)$ calculated from SANS data, $B_{\xi}$.

$B_{\eta}$ for the three samples studied. We note that since the $\eta_{s p} \propto N$ dependence predicted by eq 4 is not observed experimentally, ${ }^{20}$ $B_{\eta}$ effectively becomes $N$ dependent. This dependence is not real but simply reflects a shortcoming of the scaling model. For all three samples $B_{\eta} / B_{\xi} \simeq 2.3-3$. The values observed for other polyelectrolyte systems differ: Dou and $\mathrm{Colby}^{36}$ find $B_{\eta} / B_{\xi}=$
2.7 and $B_{\eta} / B_{\xi}=1$ for quaternized poly(2-vinylpyridine) in ethylene glycol and $N$-methylformamide, respectively; Di Cola et al. find $B_{\eta} / B_{\xi}=0.9-1.3$ for a range of maleate copolymers in aqueous solution, and data for sodium polystyrenesulfonate gives $B_{\eta} / B_{\xi} \simeq 0.5 .^{32,83,84}$ The differences observed likely arise due to the neglect of prefactors in the calculation of the Rouse and Zimm times and due to the use of the static correlation length to estimate the crossover length scale at which hydrodynamic interactions become screened. ${ }^{28}$ We consider the SANS estimate of $B$ to be more reliable as it directly probes the mesh size of polyelectrolyte solutions without making assumptions about their hydrodynamic properties.

The specific viscosities of the three samples are plotted as a function of polymer concentration in Figure 5a, and the same
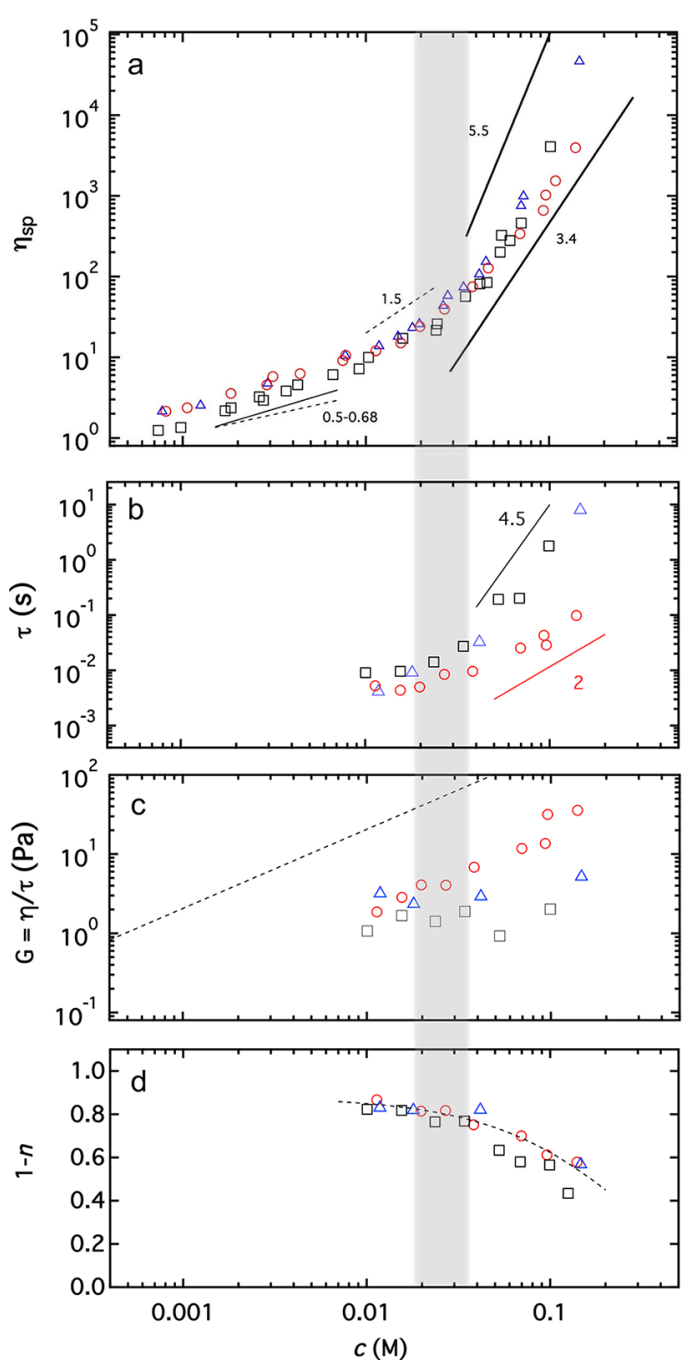

Figure 5. Comparison of rheological properties of $\mathrm{NaCMC}$ samples of different degrees of substitution in salt-free aqueous solutions. CMC1.2 (O, data from ref 21), CMC0.8 $(\triangle)$, and CMC0.7 ( $\square$ ). (a) Specific viscosity as a function of concentration. Full black lines are best fit power laws with exponents indicated on the graph. Dashed lines are scaling predictions (eqs 4 and 6). (b) Longest relaxation time as a function of polymer concentration. (c) Terminal modulus, calculated as the ratio of the viscosity to the longest relaxation time as a function of concentration. The dashed line is the scaling prediction of $k_{\mathrm{B}} T$ per chain for $N=1250$ (CMC1.2). (d) $1-n$ as a function of polymer concentration. The vertical shaded area marks the entanglement transition. 

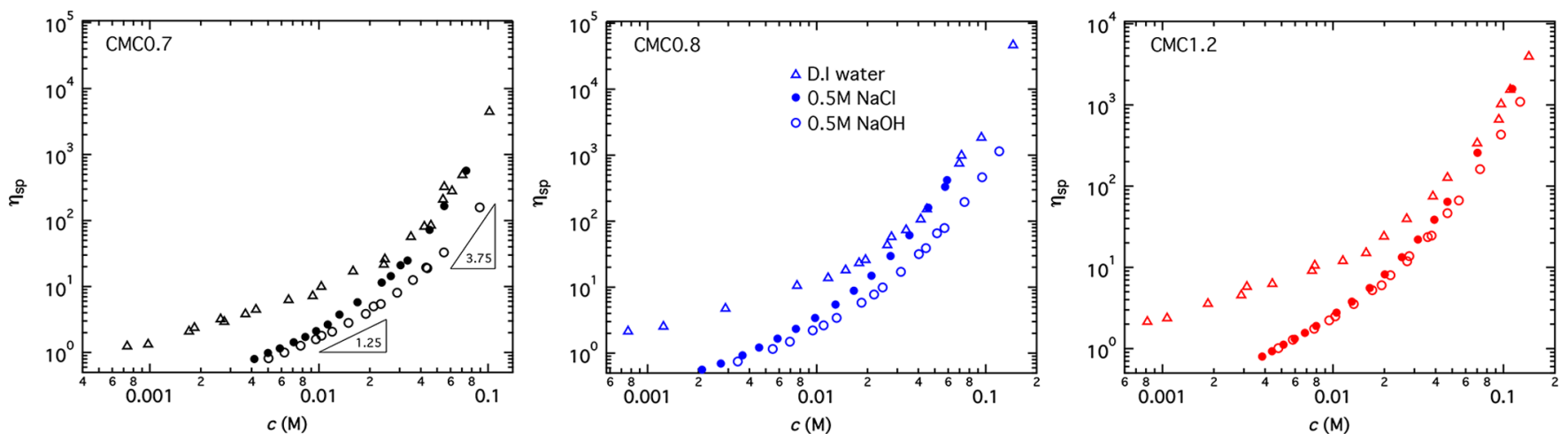

Figure 6. Specific viscosity as a function of concentration for $\mathrm{NaCMC}$ solution in DI water $(\triangle), 0.5 \mathrm{M} \mathrm{NaCl}(\mathbf{\bullet})$, and $0.5 \mathrm{M} \mathrm{NaOH}(\mathrm{O})$ for $\mathrm{NaCMC}$ with DS $=0.7,0.8$, and 1.2 as indicated in the panels. Scaling predictions for polymer in good solvent (eqs 4 and 6 for $\nu=0.59$ ) are shown for CMC0.7. Salt-free and $0.5 \mathrm{M} \mathrm{NaCl}$ for CMC1.2 data reported earlier. ${ }^{20,21}$

best fit power law exponent $(0.68 \pm 0.05)$ is observed for all samples in the semidilute unentangled regime, somewhat higher than the eq 4 value of $1 / 2$ for $\nu=1$ and in agreement with earlier results, presented in Figure S7 of ref 20. For consistency, we opt to determine the crossover concentrations $c_{\mathrm{e}}$ and $c^{* *}$ from the intersections of the predicted power laws, as shown in Figure 3. In Figure 5 we show both the best fit and predicted power laws. We note that the exact criteria used does not have a significant effect on the resulting crossovers. ${ }^{21}$

In entangled solutions, stronger power law exponents for the concentration dependence of the specific viscosity are observed for samples CMC0.7 and CMC0.8 than for CMC1.2. The longest relaxation times, estimated by $\tau$ in eq 7 and plotted in Figure $5 b$, also increase for the less substituted samples. These two observations are interpreted as resulting from associations between chains, ${ }^{65}$ which arise due to unsubstituted patches along the cellulose chain. ${ }^{49,85}$ The flow index for the three samples, plotted in Figure 5d, appears to be unaffected by hydrophobic interactions. The values of $n \simeq 0.1-0.2$ in the unentangled regime are lower than the predicted value of $n=$ 0.5 by Colby et al. ${ }^{86}$ and may reflect the limitations of our fits given the restricted experimentally accessible shear rate range.

The terminal modulus, which can be estimated as $G \simeq \eta / \tau$, follows an approximately linear relation with concentration, given by $G \simeq k_{\mathrm{B}} T c / 10 N$. The factor of $1 / 10$ is probably the result of the polydispersity of the samples and the method used to estimate the terminal modulus. While the zero-shear rate viscosity corresponds to an average over all chains, the relaxation time is identified from the onset of shear thinning and therefore corresponds to the highest molar mass fraction, yielding artificially low values of G. CMC0.7 and CMC0.8 deviate to lower values at high concentrations, which we interpret as arising due to chain association and coupled relaxation, thereby decreasing the number density of chains in solution.

We observed no change in the viscosity of a CMC0.7 solution with $c \simeq 0.05 \mathrm{M}$ after filtering through a $0.2 \mu \mathrm{m}$ size filter. Light scattering data confirm the removal of aggregates, which results in a large decrease of the scattered intensity at low angles and a decrease in the amplitude of the slow mode decay in the intensity correlation function. It appears that supramolecular structures (possibly fringed micelles) do not significantly affect the rheological behavior of $\mathrm{NaCMC}$ solutions. The increase of viscosity at higher concentrations (which we are not able to filter) probably has a different origin, namely, temporary associations between dissolved chains and/ or the formation of a polymer network.

$\mathrm{NaCl}$ and $\mathrm{NaOH}$ Solutions. Having assessed the rheology in DI water, we now turn to solutions containing excess salt $(0.5$ $\mathrm{M})$, i.e., solutions with many more added salt ions than free polyelectrolyte counterions. Under these conditions, the Debye screening length is $\kappa^{-1} \simeq 0.4 \mathrm{~nm}$, meaning electrostatic forces become short-ranged and analogous to excluded volume forces in neutral polymer solutions. ${ }^{87,88}$ The solution viscosities for which the salt was dissolved before the polymer were found to be identical to those where the salt was added to the dissolved polymer for all samples, in contrast to previous reports by Francis ${ }^{44}$ and Yang and $\mathrm{Zhu} .{ }^{64}$ We interpret this difference as due to incomplete dissolution of their samples. The electrostatic interactions are highly screened, and thus neutral polymer in good solvent behavior is observed; the viscosity varies as $\eta_{\mathrm{sp}}$ $\propto c^{1.3 \pm 0.1}$ in the semidilute unentangled regime, in good agreement with eq 4 for $\nu=0.59$.

Figure 6 compares the specific viscosity as a function of polymer concentration for the three $\mathrm{NaCMC}$ samples in DI water as well as $0.5 \mathrm{M} \mathrm{NaCl}$ and $0.5 \mathrm{M} \mathrm{NaOH}$ aqueous solutions. As expected, addition of salt decreases the solution viscosity at low concentrations for all samples and electrolytes. This behavior is the result of screening of repulsive forces between charged groups along the polymer chain ${ }^{20,28}$ which leads to reduced chain dimensions. At high concentrations, the viscosities of the salt-free solutions and the $0.5 \mathrm{M} \mathrm{NaCl}$ solutions converge as salt does not contribute to further screening.

Highly substituted CMC1.2 displays very similar solution viscosities in $\mathrm{NaCl}$ and $\mathrm{NaOH}$. For CMC0.8 and $\mathrm{CMC} 0.7$, by contrast, the viscosities in the presence of $\mathrm{NaOH}$ are significantly lower than in $\mathrm{NaCl}$ at high polymer concentrations. We interpret this decrease in viscosity in the presence of $\mathrm{NaOH}$ as resulting from the solubilization of unsubstituted cellulose blocks. ${ }^{89}$

The onset of hydrophobic interactions may be identified in the plots of $\eta_{\mathrm{sp}}$ vs $c[\eta]$ as the point where the viscosities in $\mathrm{NaCl}$ and $\mathrm{NaOH}$ diverge. This is seen to occur around $c \simeq 5-$ $10 c^{*}$ for samples CMC0.7 and CMC0.8 in Figure 7. We observe power law exponents for the viscosity dependence with concentration of $m \simeq 4.3$ for CMC1.2 and $m \simeq 5.5-7$ for samples CMC0.7 and CMC0.8 in $\mathrm{NaCl}$, compiled in Table 3. By contrast, in $\mathrm{NaOH}$, all points collapse onto the same curve, as expected for polymers with the same polymer-solvent interactions, only differing in molecular weight. ${ }^{1,43}$ 

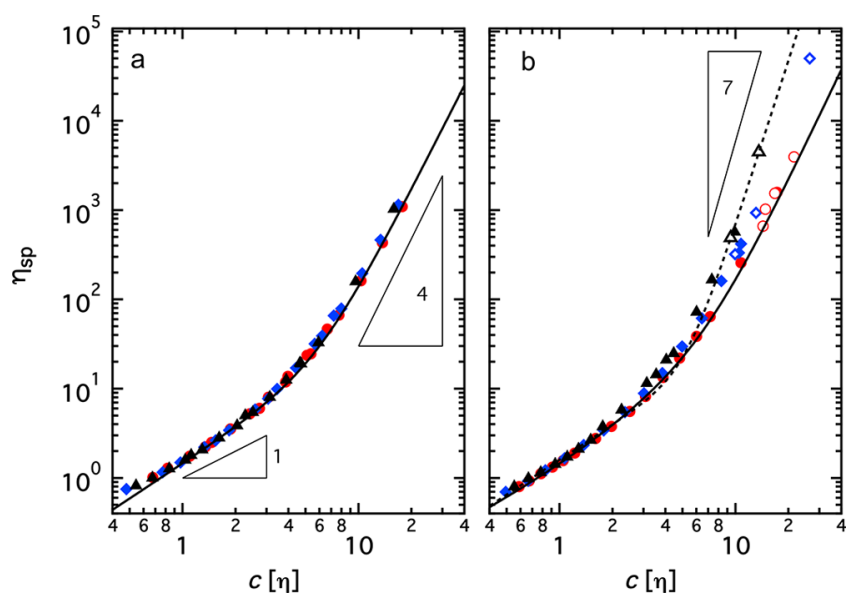

Figure 7. Specific viscosity as a function of the overlap parameter for solutions of the three NaCMC samples studied (CMC1.2: red circles; CMC0.8: blue rhombuses; CMC0.7: black triangles) in (a) $0.5 \mathrm{M}$ $\mathrm{NaOH}$. Black line is a fit to eq 9, using parameters for $\mathrm{CMC} 0.7$ given in Table 3. (b) $0.5 \mathrm{M} \mathrm{NaCl}$ (open symbols correspond to solutions in DI water, as in this range the data in $\mathrm{DI}$ and $0.5 \mathrm{M} \mathrm{NaCl}$ converge; ${ }^{20}$ the value of $[\eta]$ corresponds to the one in $\mathrm{NaCl}$ for all points) The full black line is the same as in part (a); the dotted line is the eq 9 fit to CMC0.7 data, with fit parameters in Table 3.

Table 3. Fit Parameters for Eqs 8 and 9 in $0.5 \mathrm{M} \mathrm{NaOH}^{a}$

\begin{tabular}{|c|c|c|c|c|c|}
\hline & $c_{\mathrm{e}}(\mathrm{M})$ & {$[\eta]\left(\mathrm{M}^{-1}\right)$} & $B^{\prime}$ & $m$ & $c_{\mathrm{e}} / c^{*}$ \\
\hline \multicolumn{6}{|c|}{$0.5 \mathrm{M} \mathrm{NaOH}$} \\
\hline CMC1.2 & 0.051 & 146 & $8.6 \times 10^{-3}$ & 4.0 & 7.4 \\
\hline СMC0.8 & 0.05 & 142 & $7.6 \times 10^{-3}$ & 4.2 & 7.1 \\
\hline СMC0.7 & 0.06 & 109 & $6.1 \times 10^{-3}$ & 4.3 & 6.4 \\
\hline \multicolumn{6}{|c|}{$0.5 \mathrm{M} \mathrm{NaCl}$} \\
\hline CMC1.2 & 0.036 & 152 & $2.4 \times 10^{-3}$ & 4.4 & 5.5 \\
\hline
\end{tabular}

${ }^{a}$ The Huggins coefficient was fixed at $k_{\mathrm{H}}=0.45$ for all fits to eq 9. Fits to eq 8 used exponents $\alpha=1.3$ and $\beta=2.6$ predicted by scaling theory for neutral polymers in good solvent.
Entanglement in Salt Solutions. The entanglement concentrations are obtained by fitting data above the overlap concentration to

$$
\eta_{\mathrm{sp}}=\left[\eta_{\mathrm{sp}}\left(c^{*}\right)\left(c / c^{*}\right)^{\alpha}\right]\left(1+\left(c / c_{\mathrm{e}}\right)^{\beta}\right)
$$

where the term in square brackets is the viscosity dependence predicted by the Rouse model for semidilute unentangled solutions and $\eta_{\text {sp }}\left(c^{*}\right)$ is the specific viscosity at the overlap concentration. Equation 8 interpolates eqs 4 and 6 when $\alpha=$ $1 /(3 \nu-1)$ and $\beta=2 \alpha$. Additionally, data were fitted to an expanded Huggins equation: ${ }^{1,90}$

$$
\eta_{\mathrm{sp}}=c[\eta]+k_{\mathrm{H}}(c[\eta])^{2}+B^{\prime}(c[\eta])^{m}
$$

where $k_{\mathrm{H}}$ is the Huggins coefficient, $B^{\prime}$ is a parameter related to the onset of entanglement, and $m$ gives the power law exponent of the specific viscosity with concentration in the entangled regime. The viscosity at the overlap concentration is given by $\eta_{\mathrm{sp}}\left(c^{*}\right)=1+k_{\mathrm{H}}+B^{\prime} \simeq 1+k_{\mathrm{H}}$. The parameters from fits to eqs 8 and 9 are collected in Table 3. The entanglement parameters for $\mathrm{NaOH}$ solutions are similar for all three samples.

The concentration dependence of the viscosity in entangled solution varies as $\eta_{\mathrm{sp}} \propto c^{4.2 \pm 0.2}$. The ratio of the entanglement to overlap concentration is found to be $c_{\mathrm{e}} / c^{*} \simeq 6-7$, in agreement with an earlier report in $0.1 \mathrm{M} \mathrm{NaCl} .{ }^{20}$ Computing this ratio from the compilation of $\mathrm{Heo}$ et al. for flexible, neutral polymers, ${ }^{91}$ we find $c_{\mathrm{e}} / c^{*} \simeq 3-15$. Note that we have adjusted their values to match our fitting methods. ${ }^{92}$ The similar range of values is consistent with $\mathrm{NaCMC}$ in $0.5 \mathrm{M} \mathrm{NaOH}$ behaving similarly to a neutral polymer in good solvent.

A value of the melt entanglement molecular weight $M_{\mathrm{e}} \simeq$ $3900 \mathrm{~g} / \mathrm{mol}$ was reported by Horinaka et al. ${ }^{93}$ for of $\mathrm{NaCMC}$ $(\mathrm{DS} \simeq 0.7)$ in an ionic liquid. This result, combined with the scaling prediction

$$
c_{\mathrm{e}} / c^{*} \simeq N_{\mathrm{e}}(1)^{3 \nu-1}
$$

where $N_{\mathrm{e}}(1)$ is the number of Kuhn segments ${ }^{94}$ between entanglements in the melt, ${ }^{95}$ gives $c_{\mathrm{e}} / c^{*} \simeq 1$. This value
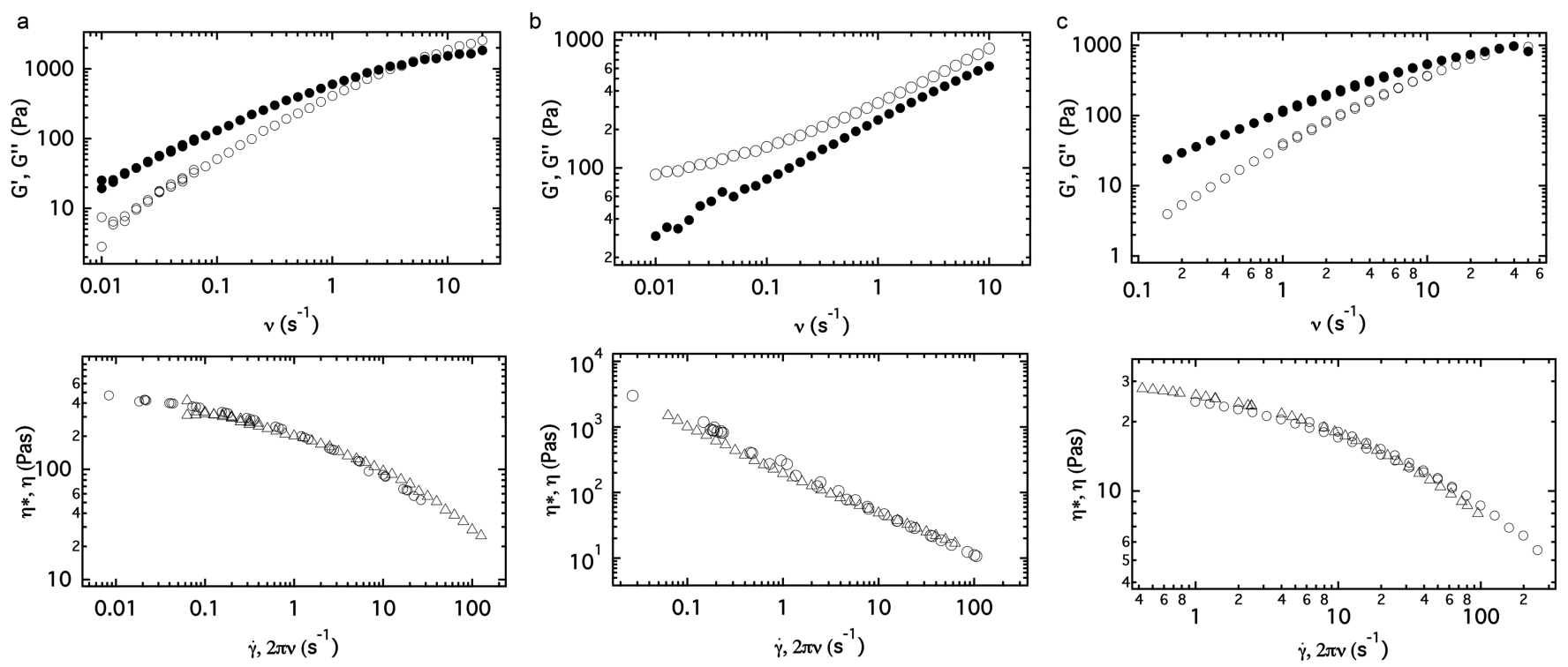

Figure 8. Oscillatory rheology data for solutions of NaCMC: (a) CMC1.2, $c \simeq 0.41 \mathrm{M}$ solution in DI water; (b) CMC0.7 $c \simeq 0.37 \mathrm{M}$ in DI water; (c) $\mathrm{CMC} 0.7 \mathrm{c} \simeq 0.37 \mathrm{M}$ in $0.5 \mathrm{M} \mathrm{NaOH}$ aqueous solution. Top panels: $(\mathbf{O})$ loss modulus $G^{\prime \prime}$, (O) storage modulus $G^{\prime}$; bottom panels: $(\bigcirc)$ complex viscosity and $(\triangle)$ steady shear viscosity. 

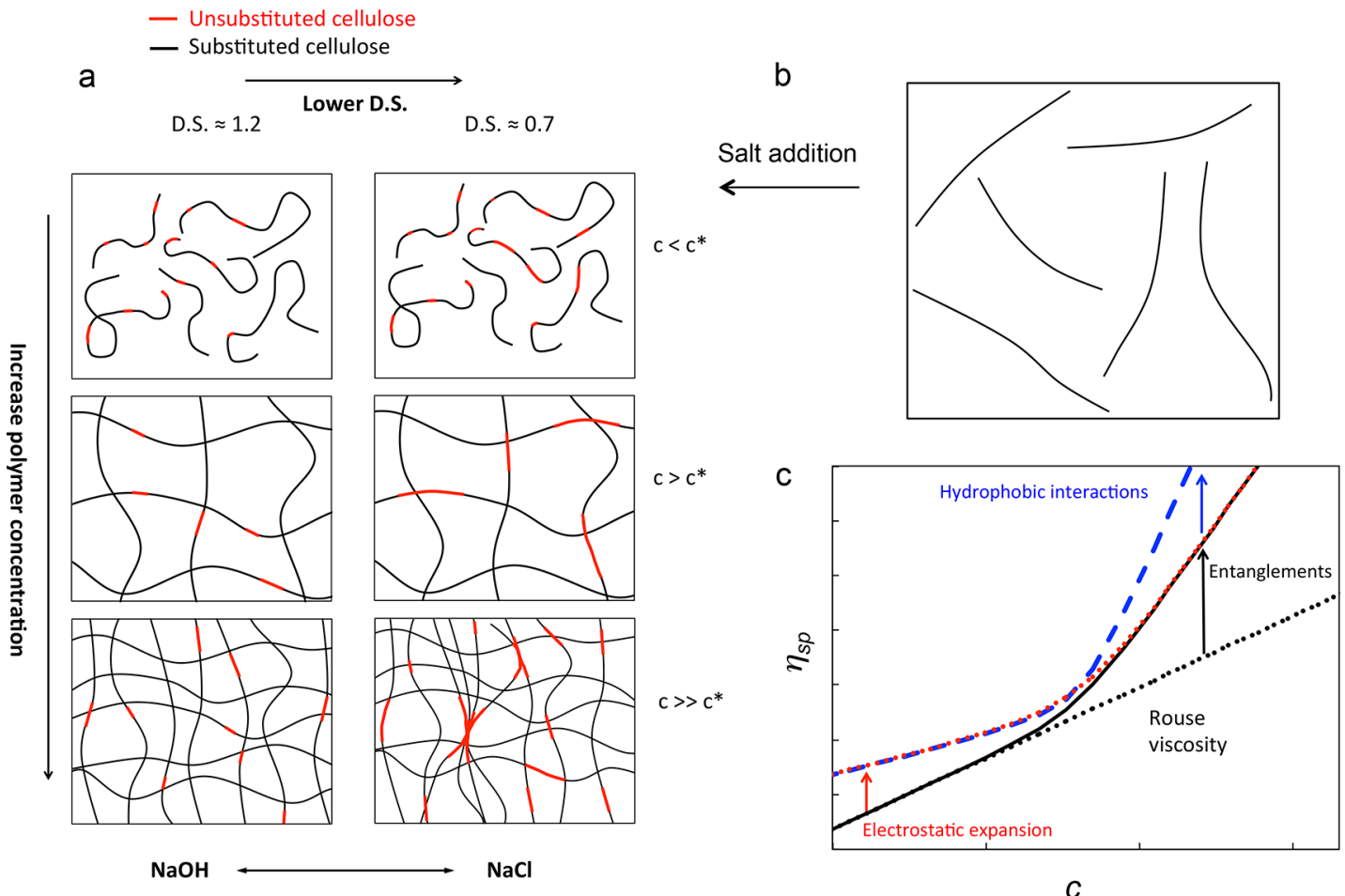

C

Figure 9. (a) Schematic of the structure of $\mathrm{NaCMC}$ solutions as a function of polymer concentration, DS and $\mathrm{pH}$. For $c \lesssim 5 c^{*}$, neither $\mathrm{NaOH}$ nor DS has a strong effect on solution structure; this is supported by the SANS data presented. At high concentrations, weakly substituted (DS $\simeq 0.7$ ) $\mathrm{NaCMC}$ contains a large number of interchain associations which lead to increased viscosities and gelation $c \gtrsim 0.2 \mathrm{M}$ in DI water. By contrast, highly substituted $\mathrm{NaCMC}$ shows standard (nonassociative) polymer behavior. Addition of $\mathrm{NaOH}$ solvates the unsubstituted cellulose backbone (red lines) and has an effect that is similar to increasing the DS. At low DS, addition of $\mathrm{NaOH}$ is sufficient to suppress association, resulting in a structure as show in the left column. (b) Schematic of highly elongated chains in dilute salt-free solution. (c) Schematic of the various contributions to the dependence of solution viscosity on polymer concentration. Black dotted line: unentangled solution viscosity with weak electrostatics. Full black line: entanglement interactions (above $c_{\mathrm{e}}$ ) with weak electrostatics (note that this line overlaps with the dotted red and black lines at high and low concentrations respectively). Red line: entanglement interactions with strong electrostatics. Blue line: entanglement interactions with strong electrostatics and hydrophobic association.

disagrees with our observations in solution. We do not at the moment have an explanation for this disagreement.

Oscillatory Rheology. The hydrophobic character of sample CMC0.7 was further quantified by oscillatory shear rheology experiments. Figure $8 \mathrm{~b}$ plots the loss and storage modulus for a solution of $\simeq 0.35 \mathrm{M}$ in DI water. The sample displays a weak gel-like behavior, with $G^{\prime} \geq G^{\prime \prime}$ over the entire frequency $\left(\nu\right.$, in $\left.\mathrm{s}^{-1}\right)$ range. We note that the observed behavior does not satisfy some common definitions of gels in that a plateau in not observed for $G^{\prime}$; additionally, $G^{\prime}$ is never much larger than $G^{\prime \prime}$. At these high concentrations, it is not possible to obtain a value for $\eta(0)$. Measurements a few days after preparation and after one year yield very similar results (Figure S9), indicating the gel structure is stable. Shearing at $100 \mathrm{~s}^{-1}$ results in a decrease in the solution viscosity and of $G^{\prime}$ (but not $\left.G^{\prime \prime}\right)$. We did not observe a recovery of the viscosity following the shearing step, which therefore does not qualify this sample as thixotropic in the time range studied $(\simeq 30 \mathrm{~min}$ after shearing), detailed in Figure S9. In $\mathrm{NaOH}$ (Figure 8c), the solution displays a lower viscosity and the variation of $G^{\prime \prime}$ and $G^{\prime}$ with oscillation frequency is characteristic of an entangled polymer solution. Solubilization of the unsubstituted cellulose blocks by $\mathrm{NaOH}$ therefore turns the gel into a solution. A $c \simeq$ $0.4 \mathrm{M} \mathrm{CMC1.2} \mathrm{solution} \mathrm{in} \mathrm{DI} \mathrm{water} \mathrm{is} \mathrm{also} \mathrm{a} \mathrm{viscoelastic} \mathrm{liquid}$ (Figure 8a), demonstrating that the hydrophobic interactions in this sample are significantly weaker, as anticipated from the concentration dependence of the viscosity. The samples studied obey the Cox-Merz rule, as shown in the bottom panels of Figure 8.

\section{CONCLUSIONS}

We have examined the effect of the degree of substitution on the structure and rheology of $\mathrm{NaCMC}$ in aqueous solutions. SANS and rheology data were interpreted in the framework of the de Gennes, Pfeuty, and Dobrynin scaling models of polyelectrolyte solutions. Despite some shortcomings, discussed throughout the text, these models allow us to identify the key crossover concentrations and resolve the contributions of different interactions to the flow behavior of $\mathrm{NaCMC}$.

Lowering DS below $\simeq 1$ is found to favor polymer-polymer interactions, resulting in hydrophobic behavior; in turn these associations cause increased viscosities and eventual gelation. The correlation length is found to be largely unaffected by DS, within experimental uncertainty, indicating that these interactions do not significantly alter, on average, chain conformation (e.g., the persistence length) or polymer solubility in the semidilute regime. Hydrophobic interactions have an increasingly large effect on the solution rheology as the polymer concentration increases.

Addition of $\mathrm{NaCl}$ screens electrostatic interactions, decreasing the solution viscosities at low polymer concentrations; at higher concentrations $(\gtrsim 0.1 \mathrm{M})$, electrostatics are largely screened by counterions and addition of $\mathrm{NaCl}$ does not contribute significantly to further screening. Addition of $\mathrm{NaOH}$ 
diminishes hydrophobic as well as electrostatic interactions, resulting in a decrease in solution viscosities at high polymer concentrations for weakly substituted samples $(0.7-0.8)$. At lower polymer concentrations, $\mathrm{NaOH}$ decreases solution viscosities by a similar amount as $\mathrm{NaCl}$. A summary of the effects of electrostatic and hydrophobic forces on $\mathrm{NaCMC}$ solutions is shown schematically in Figure 9.

Hydrophobic interactions result in a viscosity increase at high $\left(c \gtrsim 5 c^{*}\right)$ concentration. Electrostatic interactions primarily affect solution viscosity at low concentration due to repulsion, leading to chain expansion, while at high polymer concentrations these interactions are screened (even in the absence of salt). The role of entanglement is manifested at $c>c_{\mathrm{e}}$ and is not significantly affected by salt.

This work provides mechanistic insight into the rheological behavior of $\mathrm{NaCMC}$ under representative solution environments enabling quantitative control of flow properties of this ubiquitous water-soluble cellulose derivative.

\section{ASSOCIATED CONTENT}

\section{S Supporting Information}

The Supporting Information is available free of charge on the ACS Publications website at DOI: 10.1021/acs.macromol.8b00178.

Detailed analysis of the SANS data, including the low $q$ upturn; fits to different models (Carreau, Cross, Carreau-Yasuda) to shear rate dependent viscosity (PDF)

\section{AUTHOR INFORMATION}

\section{Corresponding Author}

*E-mail j.cabral@imperial.ac.uk; phone +44(0)2075945571 (J.T.C.).

ORCID

Carlos G. Lopez: 0000-0001-6160-632X

Ralph H. Colby: 0000-0002-5492-6189

João T. Cabral: 0000-0002-2590-225X

\section{Present Address}

C.G.L.: Institute of Physical Chemistry, RWTH Aachen University, Landoltweg 2, D-52056 Aachen, Germany.

Notes

The authors declare no competing financial interest.

\section{ACKNOWLEDGMENTS}

We thank the Engineering and Physical Sciences Research Council (EPSRC, UK) and Unilever for financial support, ILL and ISIS for beam time, and a Leverhulme visiting professorship to RHC. We also thank Alessandra Vitale for help with the DS determination experiments and Walter Richtering (RWTH Aachen) for access to the Kinexus rheometer. Data are available upon request: please contact polymer-microfluidics@imperial. ac.uk.

\section{REFERENCES}

(1) Clasen, C.; Kulicke, W.-M. Determination of Viscoelastic and Rheo-optical Material Functions of Water-Soluble Cellulose Derivatives. Prog. Polym. Sci. 2001, 26, 1839-1919.

(2) Zhang, Z.; Chen, Q.; Colby, R. H. Dynamics of associative polymers. Soft Matter 2018, DOI: 10.1039/C8SM00044A.

(3) Ganesan, M.; Knier, S.; Younger, J. G.; Solomon, M. J. Associative and Entanglement Contributions to the Solution Rheology of a Bacterial Polysaccharide. Macromolecules 2016, 49, 8313-8321.
(4) Kujawa, P.; Audibert-Hayet, A.; Selb, J.; Candau, F. Rheological Properties of Multisticker Associative Polyelectrolytes in Semidilute Aqueous Solutions. J. Polym. Sci., Part B: Polym. Phys. 2004, 42, 16401655.

(5) Kujawa, P.; Audibert-Hayet, A.; Selb, J.; Candau, F. Effect of Ionic Strength on the Rheological Properties of Multisticker Associative Polyelectrolytes. Macromolecules 2006, 39, 384-392.

(6) Goycoolea, F.; Morris, E.; Gidley, M. Viscosity of Galactomannans at Alkaline and Neutral pH: Evidence of 'Hyperentanglement' in Solution. Carbohydr. Polym. 1995, 27, 69-71.

(7) Doyle, J. P.; Lyons, G.; Morris, E. R. New Proposals on 'Hyperentanglement' of Galactomannans: Solution Viscosity of Fenugreek Gum Under Neutral and Alkaline Conditions. Food Hydrocolloids 2009, 23, 1501-1510.

(8) Boucard, N.; David, L.; Rochas, C.; Montembault, A.; Viton, C.; Domard, A. Polyelectrolyte Microstructure in Chitosan Aqueous and Alcohol Solutions. Biomacromolecules 2007, 8, 1209-1217.

(9) Popa-Nita, S.; Rochas, C.; David, L.; Domard, A. Structure of Natural Polyelectrolyte Solutions: Role of the Hydrophilic/Hydrophobic Interaction Balance. Langmuir 2009, 25, 6460-6468.

(10) Esquenet, C.; Buhler, E. Phase Behavior of Associating Polyelectrolyte Polysaccharides. 1. Aggregation Process in Dilute Solution. Macromolecules 2001, 34, 5287-5294.

(11) Esquenet, C.; Terech, P.; Boué, F.; Buhler, E. Structural and Rheological Properties of Hydrophobically Modified Polysaccharide Associative Networks. Langmuir 2004, 20, 3583-3592.

(12) Essafi, W.; Raissi, W.; Abdelli, A.; Bouè, F. Metastability of Large Aggregates and Viscosity, and Stability of The Pearl Necklace Conformation After Organic Solvent Treatment Of Aqueous Hydrophobic Polyelectrolyte Solutions. J. Phys. Chem. B 2014, 118, 1227112281.

(13) Burchard, W. Structure Formation by Polysaccharides in Concentrated Solution. Biomacromolecules 2001, 2, 342-353.

(14) Essafi, W.; Spiteri, M.-N.; Williams, C.; Boué, F. Hydrophobic Polyelectrolytes in Better Polar Solvent. Structure and Chain Conformation As Seen by SAXS and SANS. Macromolecules 2009, 42, 9568-9580.

(15) Essafi, W.; Haboubi, N.; Williams, C.; Boué, F. Weak Temperature Dependence of Structure in Hydrophobic Polyelectrolyte Aqueous Solution (PSSNa): Correlation Between Scattering and Viscosity. J. Phys. Chem. B 2011, 115, 8951-8960.

(16) Essafi, W.; Abdelli, A.; Bouajila, G.; Boué, F. Behavior of Hydrophobic Polyelectrolyte Solution in Mixed Aqueous/Organic Solvents Revealed by Neutron Scattering and Viscosimetry. J. Phys. Chem. B 2012, 116, 13525-13537.

(17) Varshney, V.; Naithani, S. Cellulose Fibers: Bio-and Nano-Polymer Composites; Springer: 2011; pp 43-60.

(18) Schulz, L.; Seger, B.; Burchard, W. Structures of Cellulose in Solution. Macromol. Chem. Phys. 2000, 201, 2008-2022.

(19) Industrial Gums: Polysaccharides and Their Derivatives, 3rd ed.; Academic Press: 1993.

(20) Lopez, C. G.; Colby, R. H.; Graham, P.; Cabral, J. T. Viscosity and Scaling of Semiflexible Polyelectrolyte $\mathrm{NaCMC}$ in Aqueous Salt Solutions. Macromolecules 2017, 50, 332-338.

(21) Lopez, C. G.; Rogers, S. E.; Colby, R. H.; Graham, P.; Cabral, J. T. Structure of Sodium Carboxymethyl Cellulose Aqueous Solutions: A SANS and Rheology Study. J. Polym. Sci., Part B: Polym. Phys. 2015, 53, 492-501.

(22) Kulicke, W.-M.; Kull, A. H.; Kull, W.; Thielking, H.; Engelhardt, J.; Pannek, J.-B. Characterization of Aqueous Carboxymethylcellulose Solutions in Terms of Their Molecular Structure and its Influence on Rheological Behaviour. Polymer 1996, 37, 2723-2731.

(23) Gibis, M.; Schuh, V.; Allard, K.; Weiss, J. Influence of Molecular Weight and Degree of Substitution of Various Carboxymethyl Celluloses on Unheated and Heated Emulsion-type Sausage Models. Carbohydr. Polym. 2017, 159, 76-85.

(24) Glasser, W.; Atalla, R.; Blackwell, J.; Malcolm Brown, J. R.; Burchard, W.; French, A.; Klemm, D.; Nishiyama, Y. About the 
Structure of Cellulose: Debating the Lindman Hypothesis. Cellulose 2012, 19, 589-598.

(25) Medronho, B.; Romano, A.; Miguel, M. G.; Stigsson, L.; Lindman, B. Rationalizing Cellulose (in) Solubility: Reviewing Basic Physicochemical Aspects and Role of Hydrophobic Interactions. Cellulose 2012, 19, 581-587.

(26) Ray, D.; De, R.; Das, B. Thermodynamic, Transport and Frictional Properties in Semidilute Aqueous Sodium Carboxymethylcellulose Solution. J. Chem. Thermodyn. 2016, 101, 227-235.

(27) Hoogendam, C. W.; de Keizer, A.; Cohen Stuart, M. A.; Bijsterbosch, B. H.; Smit, J. A. M.; van Dijk, J. A. P. P.; van der Horst, P. M.; Batelaan, J. G. Persistence Length of Carboxymethyl Cellulose As Evaluated from Size Exclusion Chromatography and Potentiometric Titrations. Macromolecules 1998, 31, 6297-6309.

(28) Dobrynin, A. V.; Colby, R. H.; Rubinstein, M. Scaling Theory of Polyelectrolyte Solutions. Macromolecules 1995, 28, 1859-1871.

(29) Salamon, K.; Aumiler, D.; Pabst, G.; Vuletić, T. Probing the Mesh Formed by the Semirigid Polyelectrolytes. Macromolecules 2013, 46, 1107-1118.

(30) Papagiannopoulos, A.; Sotiropoulos, K.; Radulescu, A. Scattering Investigation of Multiscale Organization in Aqueous Solutions of Native Xanthan. Carbohydr. Polym. 2016, 153, 196-202.

(31) Wolf, B. A. Polyelectrolytes Revisited: Reliable Determination of Intrinsic Viscosities. Macromol. Rapid Commun. 2007, 28, 164-170.

(32) Boris, D. C.; Colby, R. H. Rheology of Sulfonated Polystyrene Solutions. Macromolecules 1998, 31, 5746-5755.

(33) Colby, R. H.; Rubinstein, M. Two-Parameter Scaling for Polymers in $\theta$ Solvents. Macromolecules 1990, 23, 2753-2757.

(34) Wang, L.; Bloomfield, V. A. Osmotic Pressure of Polyelectrolytes Without Added Salt. Macromolecules 1990, 23, 804-809.

(35) Lorchat, P.; Konko, I.; Combet, J.; Jestin, J.; Johner, A.; Laschewski, A.; Obukhov, S.; Rawiso, M. New Regime in Polyelectrolyte Solutions. EPL 2014, 106, 28003.

(36) Dou, S.; Colby, R. H. Charge Density Effects in Salt-Free Polyelectrolyte Solution Rheology. J. Polym. Sci., Part B: Polym. Phys. 2006, 44, 2001-2013.

(37) Takahashi, Y.; Iio, S.; Matsumoto, N.; Noda, I. Viscoelastic properties of polyelectrolyte solutions in non-entangled concentrated regions. Polym. Int. 1996, 40, 269-273.

(38) Takahashi, Y.; Matsumoto, N.; Iio, S.; Kondo, H.; Noda, I.; Imai, M.; Matsushita, Y. Concentration Dependence of Radius of Gyration of Sodium Poly(styrenesulfonate) over a Wide Range of Concentration Studied by Small-Angle Neutron Scattering. Langmuir 1999, 15, 4120-4122.

(39) Guillot, S.; Delsanti, M.; Desert, S.; Langevin, D. SurfactantInduced Collapse of Polymer Chains and Monodisperse Growth of Aggregates near the Precipitation Boundary in Carboxymethylcellulose-DTAB Aqueous Solutions. Langmuir 2003, 19, 230-237.

(40) Tam, K.; Tiu, C. Improved Correlation for Shear-Dependent Viscosity of Polyelectrolyte Solutions. J. Non-Newtonian Fluid Mech. 1993, 46, 275-288.

(41) Wu, Q.; Shangguan, Y.; Du, M.; Zhou, J.; Song, Y.; Zheng, Q. Steady and dynamic rheological behaviors of sodium carboxymethyl cellulose entangled semi-dilute solution with opposite charged surfactant dodecyl-trimethylammonium bromide. J. Colloid Interface Sci. 2009, 339, 236-242.

(42) Różańska, S.; Różański, J. Extensional Flow of Carboxymethylcellulose Sodium Salt Measured on the Opposed-Nozzle Device. Soft Mater. 2017, 15, 302-314.

(43) Colby, R. H. Structure and Linear Viscoelasticity of Flexible Polymer Solutions: Comparison of Polyelectrolyte and Neutral Polymer Solutions. Rheol. Acta 2010, 49, 425-442.

(44) Francis, P. S. Solution Properties of Water-Soluble Polymers. I. Control of Aggregation of Sodium Carboxymethylcellulose (CMC) by Choice of Solvent and/or Electrolyte. J. Appl. Polym. Sci. 1961, 5, 261270.

(45) deButts, E. H.; Hudy, J. A.; Elliott, J. H. Rheology of Sodium Carboxymethylcellulose Solutions. Ind. Eng. Chem. 1957, 49, 94-98.
(46) Elliot, J.; Ganz, A. Some Rheological Properties of Sodium Carboxymethylcellulose Solutions and Gels. Rheol. Acta 1974, 13, 670-674.

(47) Barba, C.; Montané, D.; Rinaudo, M.; Farriol, X. Synthesis and characterization of carboxymethylcelluloses (CMC) from non-wood fibers I. Accessibility of cellulose fibers and CMC synthesis. Cellulose 2002, 9, 319-326.

(48) Lim, S.; Kim, S.; Ahn, K. H.; Lee, S. J. The effect of binders on the rheological properties and the microstructure formation of lithiumion battery anode slurries. J. Power Sources 2015, 299, 221-230.

(49) Enebro, J.; Momcilovic, D.; Siika-Aho, M.; Karlsson, S. A New Approach for Studying Correlations Between the Chemical Structure and the Rheological Properties in Carboxymethyl Cellulose. Biomacromolecules 2007, 8, 3253-3257.

(50) Li, B.; Shao, Z.-Q.; Hong, J.-M.; Wang, F.-J.; Zhang, Y.-D.; Liao, B. Effects of Dispersed Medium Systems on Substitution Pattern and Solution Performance of Carboxymethyl cellulose. Frontiers of Materials Science in China 2010, 4, 306-313.

(51) Ott, E.; Elliott, J. H. Observations on the Thixotropy and Structural Characteristics of Sodium Carboxymethylcellulose. Makromol. Chem. 1956, 18, 352-366.

(52) Dolz, M.; Bugaj, J.; Pellicer, J.; Hernandez, M.; Gorecki, M. Thixotropy of Highly Viscous Sodium (Carboxymethyl)Cellulose Hydrogels. J. Pharm. Sci. 1997, 86, 1283-1287.

(53) Ghannam, M. T.; Esmail, M. N. Rheological Properties of Carboxymethyl Cellulose. J. Appl. Polym. Sci. 1997, 64, 289-301.

(54) Edali, M.; Esmail, M. N.; Vatistas, G. H. Rheological Properties of High Concentrations of Carboxymethyl Cellulose Solutions. J. Appl. Polym. Sci. 2001, 79, 1787-1801.

(55) Karataş, M.; Arslan, N. Flow Behaviours of Cellulose and Carboxymethyl Cellulose from Grapefruit Peel. Food Hydrocolloids 2016, 58, 235-245.

(56) Hermans, J. Investigation of the Elastic Properties of the Particle Network in Gelled Solutions of Hydrocolloids. I. Carboxymethyl Cellulose. J. Polym. Sci., Part A: Gen. Pap. 1965, 3, 1859-1868.

(57) Savadkoohi, S.; Farahnaky, A. Small Deformation Viscoelastic and Thermal Behaviours of Pomegranate Seed Pips CMC Gels. J. Food Sci. Technol. 2015, 52, 4186-4195.

(58) Kastner, U.; Hoffmann, H.; Donges, R.; Hilbig, J. Structure and Solution Properties of Sodium Carboxymethyl Cellulose. Colloids Surf., A 1997, 123-124, 307-328.

(59) Meyer, F. Korrelation Rheo-Mechanischer und Rheo-Optischer Materialfunktionen. Ph.D. Thesis, Universität Hamburg, 2008.

(60) Komorowska, P.; Różańska, S.; Różański, J. Effect of the degree of substitution on the rheology of sodium carboxymethylcellulose solutions in propylene glycol/water mixtures. Cellulose 2017, 24, $4151-4162$.

(61) Kulicke, W.-M.; Reinhardt, U.; Fuller, G. G.; Arendt, O. Characterization of the Flow Properties of Sodium Carboxymethylcellulose Via Mechanical and Optical Techniques. Rheol. Acta 1999, $38,26-33$.

(62) Molecular weights calculated from the intrinsic viscosities in 5\% $\mathrm{NaOH}$ using the relation from ref 63 . The viscosities of $\mathrm{NaCMC}$ are known to not vary significantly with electrolyte concentration above $c_{\mathrm{S}}$ $\simeq 0.1 \mathrm{M},{ }^{20}$ as expected when the screening length becomes smaller than the intrinsic persistence length.

(63) Eremeeva, T.; Bykova, T. SEC of Mono-Carboxymethyl Cellulose (CMC) in a Wide Range of $\mathrm{pH}$; Mark-Houwink Constants. Carbohydr. Polym. 1998, 36, 319-326.

(64) Yang, X. H.; Zhu, W. L. Viscosity Properties of Sodium Carboxymethylcellulose Solutions. Cellulose 2007, 14, 409-417.

(65) Semenov, A.; Rubinstein, M. Dynamics of Entangled Associating Polymers with Large Aggregates. Macromolecules 2002, 35, 48214837.

(66) Rubinstein, M.; Semenov, A. N. Dynamics of Entangled Solutions of Associating Polymers. Macromolecules 2001, 34, 10581068. 
(67) Waigh, T. A.; Ober, R.; Williams, C. E.; Galin, J.-C. Semidilute and Concentrated Solutions of a Solvophobic Polyelectrolyte in Nonaqueous Solvents. Macromolecules 2001, 34, 1973-1980.

(68) Nishida, K.; Kaji, K.; Kanaya, T. Charge Density Dependence of Correlation Length Due to Electrostatic Repulsion in Polyelectrolyte Solutions. Macromolecules 1995, 28, 2472-2475.

(69) Geissler, E.; Hecht, A.-M.; Horkay, F. Scaling Behavior of Hyaluronic Acid in Solution with Mono- and Divalent Ions. Macromol. Symp. 2010, 291-292, 362-370.

(70) Horkay, F.; Basser, P. J.; Hecht, A.-M.; Geissler, E. Chondroitin sulfate in solution: Effects of Mono-and Divalent Salts. Macromolecules 2012, 45, 2882-2890.

(71) Ermi, B. D.; Amis, E. J. Influence of Backbone Solvation on Small Angle Neutron Scattering from Polyelectrolyte Solutions. Macromolecules 1997, 30, 6937-6942.

(72) Ermi, B. D.; Amis, E. J. Domain Structures in Low Ionic Strength Polyelectrolyte Solutions. Macromolecules 1998, 31, 73787384.

(73) Ermi, B. D.; Amis, E. J. Model Solutions for Studies of Salt-free Polyelectrolytes. Macromolecules 1996, 29, 2701-2703.

(74) Chremos, A.; Douglas, J. F. Counter-ion Solvation and Anomalous Low-Angle Scattering in Salt-free Polyelectrolyte Solutions. J. Chem. Phys. 2017, 147, 241103.

(75) Sedlak, M. The Ionic Strength Dependence of the Structure and Dynamics of Polyelectrolyte Solutions as Seen by Light Scattering: The Slow Mode Dilemma. J. Chem. Phys. 1996, 105, 10123-10133.

(76) Sedlak, M. Generation of Multimacroion Domains in Polyelectrolyte Solutions by Change of Ionic Strength or $\mathrm{pH}$ (Macroion Charge). J. Chem. Phys. 2002, 116, 5256-5262.

(77) Muthukumar, M. Ordinary-Extraordinary Transition in Dynamics of Solutions of Charged Macromolecules. Proc. Natl. Acad. Sci. U. S. A. 2016, 113, 12627-12632.

(78) Liebert, T. F.; Heinze, T. J. Exploitation of Reactivity and Selectivity in Cellulose Functionalization Using Unconventional Media for the Design of Products Showing New Superstructures. Biomacromolecules 2001, 2, 1124-1132.

(79) Schulz, L.; Burchard, W.; Donges, R. Cellulose Derivatives; Chapter 17, pp 218-238.

(80) Kötz, J.; Bogen, I.; Heinze, T.; Heinze, U.; Kulicke, W.-M.; Lange, S. Peculiarities in the Physico-Chemical Behaviour of NonStatistically Substituted Carboxymethylcelluloses. Colloids Surf., A 2001, 183-185, 621-633.

(81) Burchard, W. Solubility and Solution Structure of Cellulose Derivatives. Cellulose 2003, 10, 213-225.

(82) Since we do not observe the dependence of $\eta_{\mathrm{sp}} \propto c^{1 / 2}$ but rather $\eta_{\text {sp }} \sim c^{2 / 3}$, we calculate $B_{\eta}$ from an average over all unentangled data points.

(83) Kaji, K.; Urakawa, H.; Kanaya, T.; Kitamaru, R. Phase Diagram of Polyelectrolyte Solutions. J. Phys. (Paris) 1988, 49, 993-1000.

(84) Di Cola, E.; Plucktaveesak, N.; Waigh, T. A.; Colby, R. H.; Tan, J. S.; Pyckhout-Hintzen, W.; Heenan, R. K. Structure and Dynamics in Aqueous Solutions of Amphiphilic Sodium Maleate-Containing Alternating Copolymers. Macromolecules 2004, 37, 8457-8465.

(85) Barba, C.; Montané, D.; Farriol, X.; Desbrières, J.; Rinaudo, M. Synthesis and Characterization of Carboxymethylcelluloses from NonWood Pulps II. Rheological Behavior of CMC in Aqueous Solution. Cellulose 2002, 9, 327-335.

(86) Colby, R.; Boris, D.; Krause, W.; Dou, S. Shear Thinning of Unentangled Flexible Polymer Liquids. Rheol. Acta 2007, 46, 569575.

(87) Pfeuty, P. Conformation des Polyelectrolytes Ordre Dans les Solutions de Polyelectrolytes. J. Phys. Colloq. 1978, 39, C2-149-C2160.

(88) Odijk, T.; Houwaart, A. C. On the Theory of the ExcludedVolume Effect of a Polyelectrolyte in a 1-1 Electrolyte Solution. J. Polym. Sci., Polym. Phys. Ed. 1978, 16, 627-639.

(89) Alternatively, it could be argued that increasing the $\mathrm{pH}$ leads to a increased charge density along the CMC backbone with prevents hydrophobic associations. Since samples with $\mathrm{DS} \simeq 0.7$ are known to be beyond the condensation threshold in DI water, further charging is therefore unlikely to occur.

(90) Kulicke, W.-M.; Kniewske, R. The Shear Viscosity Dependence on Concentration, Molecular Weight, and Shear Rate of Polystyrene Solutions. Rheol. Acta 1984, 23, 75-83.

(91) Heo, Y.; Larson, R. G. The Scaling of Zero-Shear Viscosities of Semidilute Polymer Solutions with Concentration. J. Rheol. 2005, 49, $1117-1128$.

(92) Heo et al. ${ }^{91}$ calculate $\eta_{\text {Rouse }}$ in a manner similar to ours but use a different procedure to estimate $c_{\mathrm{e}}$. Equation 8 gives $\eta_{\mathrm{sp}}\left(c_{\mathrm{e}}\right)=2 \eta_{\text {Rouse }}$ regardless of $\alpha$ and $\beta$. We therefore estimate from Figure 4 of ref 91 that $c_{\mathrm{e}}$ using our method corresponds to a value $\simeq \times 5$ lower than their method. The ratio of entanglement to overlap concentration is then computed as $c_{\mathrm{e}}[\eta]_{0}$ in terms of the notation of their paper.

(93) Horinaka, J.-i.; Chen, K.; Takigawa, T. Entanglement Properties of Carboxymethyl Cellulose and Related Polysaccharides. Rheol. Acta 2018, 57, 51-56.

(94) We assume a Kuhn length of $10 \mathrm{~nm}$.

(95) Rubinstein, M.; Colby, R. H. Polymer Physics; Oxford University Press: 2003. 